\title{
Diversity of medicinal plants in Haluaghat upazila of Mymensingh and their uses
}

\author{
Mohammad Rizwanul Bari ${ }^{1}$, Md. Aslam Ali ${ }^{2}$ Md. Giashuddin Miah ${ }^{3}$ and Md. Rishad \\ Abdullah $^{1}$
}

\begin{abstract}
Dept. of Agrofestry, Bangladesh Agriculture University, Mymensingh, Bangladesh
${ }^{2}$ Dept. of Environmental Sciences, Bangladesh Agriculture University, Mymensingh, Bangladesh, ${ }^{3}$ Dept. of Agroforestry, Bangabandhu Sheikh Mujibur Rahman Agricultural University, Gazipur, Bangladesh
\end{abstract}

$\triangle$ For any information: ask.author@journalbinet.com, Received: 07.08.17, Revised: 26.09.17;

Available online: 14 October 2017.

\begin{abstract}
To determine the diveisty and uses of medicinal plants the study was conducted at twenty villages Haluaghat upazila of Mymensingh district, Bangladesh. Seventy five households were randomly selected and data were collected using structured questionnaires and resulted in the documentation of 87 useful medicinal plant species belonging to 51 families including trees (40\%), herbs (32\%), shrubs (20\%) and climber (8\%). The Fabaceae and Zingiberaceae family provided the largest number of species (5 plants) followed by Apocynaceae, Combretaceae and Euphorbiaceae (4 plants from each family). Among the identified plants 80 species uses against human disease, 11 species used against animal disease and 10 species used for pest control. Leaf was used in the majority of cases for medicinal preparation (52 species), followed by fruits (21 species), roots (19 species), seeds (13 species), barks (15 species) and besides these, rhizome, flower, latex as well as whole plant were used. Unfortunately, medicinal plants are increasingly threatened by various environmental, socio-economic and institutional problems. In the study area some medicinal plant species (Neem, Khejur, Mandar, Ulot kambal, Sazna, Bael and Amloki) were found well adapted for many years. However, proper training with adequate supply of desired medicinal plant species are required and credit facilities would be helpful for large scale cultivation of medicinal plants.
\end{abstract}

Key Words: Haluaghat, Medicinal plants, Diversity, Disease and Pest control

Cite Article: Bari, M. R., Ali, M. A., Miah, M. G. and Abdullah, M. R., (2017). Diversity of medicinal plants in Haluaghat upazila of Mymensingh and their uses. Journal of Bioscience and Agriculture Research, 15(02), 1266-1273. Crossref: https://doi.org/10.18801/jbar.150217.156

Article distributed under terms of a Creative Common Attribution 4.0 International License.

\section{Introduction}

Forest and village thickets have always been the major sources of medicinal plant genetic resources. In addition to the timber value, these forests are composed many medicinal and aromatic species of 
plants of diverse habits extending from trees and shrubs to herbs and climbers. Chowdhury et al. (1996) documented 42 folk formularies, which had long been used traditionally against dysentery and diarrhoea in Bangladesh. Infact, the use of plant parts for treating against diseases can be traced to the remote past and still proves to be an indispensable source of medicinal preparation, both preventive and curative (Srivastava et al., 1995). Although modern medicinal science has developed to a great extent, many rural people of this country still depend on plant products and herbal remedies for treating their ailments. Medicinal plants are playing significant role in providing primary health care services to the people. Given the importance of herbal medicines, the Government of Bangladesh has brought the system of Unani and Ayurvedic medicine under the National Drug Policy in 1982 to ensure availability, commercial manufacturing and marketing of Unani and Ayurvadic medicine and drugs (Ahsan et al., 1997). According to the World Health Organization (WHO), medicinal plants form the basis of traditional and indigenous health care needs used by the majority of the world's population. Modern Pharmacopoeia still contains at least 25\% drugs derived from plants, which are synthetic analogues built on prototype compounds isolated from plants (Silva, 1997). Medicinal plants have been used in Bangladesh in Ayurvedic and folk medicine since ancient times. Rashid et al. (1987) has conducted research work into the nature and functional dynamics of crude drug market in Bangladesh. They observed that different Ayurvedic, Unani and other pharmaceutical industries of the country commonly used some 142 different crude drugs.

About $90 \%$ of the total medicines come from plant kingdom (Rahman, 2003). From the ancient era, plants have been used for treatment of several diseases. According to experts, the herbal plants belonging to 60 families out of 206 are used for medicinal purposes in Bangladesh (Roy, 2001). In many parts of the country particularly in adjoining forest and hilly area like Haluaghat are the wild collection of medicinal species used mainly by the poor in their livelihood activity and often a major source of cash income for these groups. Most of the people of rural areas go to the kaviraj, even to Homeopathic or Allopathic doctors. Protection and conservation of medicinal plant resources were not considered as a major concern until 1984 (Hannah and Bowles, 1995). In the past, many countries have failed to adequately enforce on genetic resources of medicinal plants due to lack of awareness of the potential value of the species (Walden, 1995). From the conservation point of view, many of our traditional medicinal plants are now extinct. Some are endangered and some are totally threatened. Therefore, intensive studies on the identification and uses of medicinal plants across the country are needed for developing long term strategies for protection and conservation of medicine. By considering the above facts, the study was undertaken to identify the commonly used medicinal plant species with their diversity and uses.

\section{Materials and Methods}

The survey was conducted in twenty villages of seven union, Haluaghat upazila, Mymensingh district covering an area of $360 \mathrm{sq} \mathrm{km}$. This upazila consists of 12 union parisads, 145 mouzas and 169 villages. Total number of farm families in these villages was 59,191. Seven unions i.e. Amtail, Bhubankura, Gazirbhita, Haluaghat, Jugli, Narail and Swadeshi were selected purposively from a total of 12 unions. A total of 20 villages were taken from these seven unions for survey by the help of Upazila Agriculture Extension Office of DAE, World Vision and local people of Haluaghat. Data were collected during September to October 2004. The site is situated in the near foothill of the Garopahar and plain land that's have a long tradition of growing different medicinal plants naturally and in social and community based as well. Seventy-five respondents were chosen chiefly on the basis of knowledge on medicinal plants and their utilization in health care services and interviewed to collect data on medicinal plants and their uses. In order to collect relevant information, interview schedule was carefully designed keeping the objectives of the study in view. It contained both opened and closed form of questions. Initially the questionnaire was designed in Bengali for easy recording with the respondents. Collected data was verified through surveying the homestead and discussing with the households. Identification of the existing medicinal trees, shrubs and herbs species was done in the field with the help of rural aged people, local traditional doctors (kabiraj) while the others that could not be readily identified were brought to the herbarium of department of agroforstry, Bangladesh Agricultural University. The scientific names were obtained by consulting the literature (BARC, 19721992; Chopra et al., 1992; Chevallier, 1996; Das and Alam, 2001). The collected data were placed on to computer to prepare a database through Microsoft Excel and Microsoft Access database programme. 


\section{Results and Discussion}

A wide variety of medicinal plants were observed in the study area of Haluaghat upazila under Mymensingh district. A total of 80 plant species including herbs, shrubs, climbers and trees were frequently used by the local communities of haluaghat upzila for curing ailments were recorded during the survey (Table 01). The study revealed that tree were dominant (40\%) followed by herbs (32\%), shrubs (20\%), and climbers (8\%) (Figure 01). A similar trend was also observed by Miah and Chowdhury (2003). They found that trees were dominant on a conservation area of Northern Bangladesh and Mro tribe in Chittagong Hill Tracts, Bangladesh respectively but Ghani (2003) found that herbs were domonated on other communities of Bangladesh.

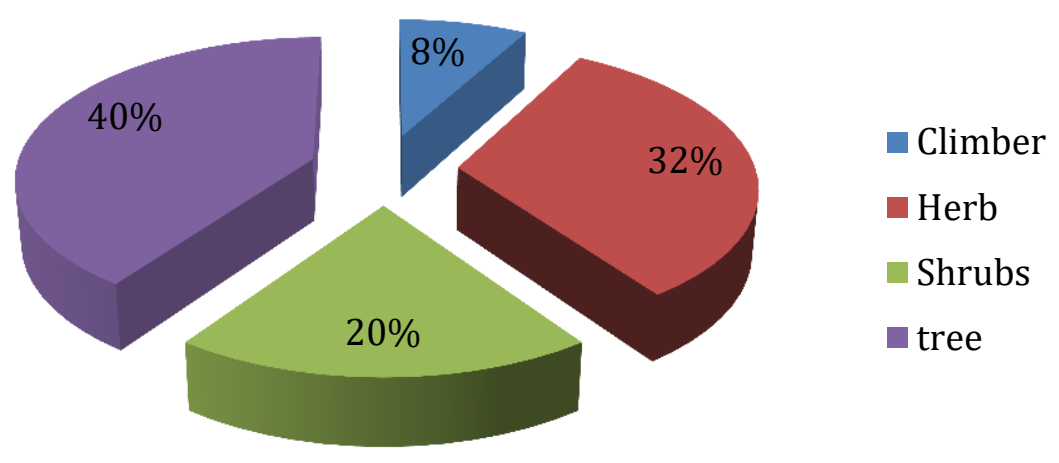

Figure 01. Percentage of type of the medicinal plants recorded from the study area.

Collected medicinal plants were distributed in 51 families. The Fabaceae and Zingiberaceae family provided the largest number of species ( 5 plants) followed by Apocynaceae, Combretaceae and Euphorbiaceae families provide 4 plants. The Amaranthaceae, Arecaceae, Malvaceae, Moraceae, Rubiaceae and Solanaceae families provide 3 plants each. The Araceae, Asteraceae, Lamiaceae, Leguminosae, Liliaceae, Menispermaceae, Rutaceae, and Verbenaceae families provided 2 plants each. Rest of the families provided only one plant. Similar trends was also obeseved by Zohora et al (2016). Plants were obtained from both wild and cultivated area. Several plants were cultivated for home consumption of fruits; the fruits were also sold commercially. Most threatened and endangered common and indigenous medicinal plant species in that locale were Horitoki, Bohera, Nishinda, Ashok, Bhuikumri, Jasthi-modhu, Anantamul, Shatamul, Datura etc.

\section{Uses of plant parts for ailment of human diseases}

Mostly of them were used against human diseases. The useful parts of the medicinal plants varied from roots to seeds. In case of some species almost all parts of that plant such as root, leaf, flower, fruit, seed etc. were found to be used against specific diseases. Total 144 uses were found in the present survey, where leaves formed the most frequently used plant part (37.96\%), followed by fruits (15.33\%), roots (13.87\%), barks (10.95\%), seeds (9.49\%), flowers (6.57\%) and rhizomes (Figure 2). The percentage of whole plant used in treatment was $2.19 \%$. For human ailments, whole plant or plant parts were either orally administered or topically applied. It was also observed that the particular plant parts are used by the practitioners in several ways. Ahmmed et. al. (2017) found 160 uses, where leaves formed the most frequently used plant part $(26.87 \%)$, followed by fruits $(18.12 \%)$, seeds $(16.25 \%)$, roots $(13.75 \%)$, stems (11.25\%), flowers (6.25\%), barks (5\%) and partial plant (3.5\%). 


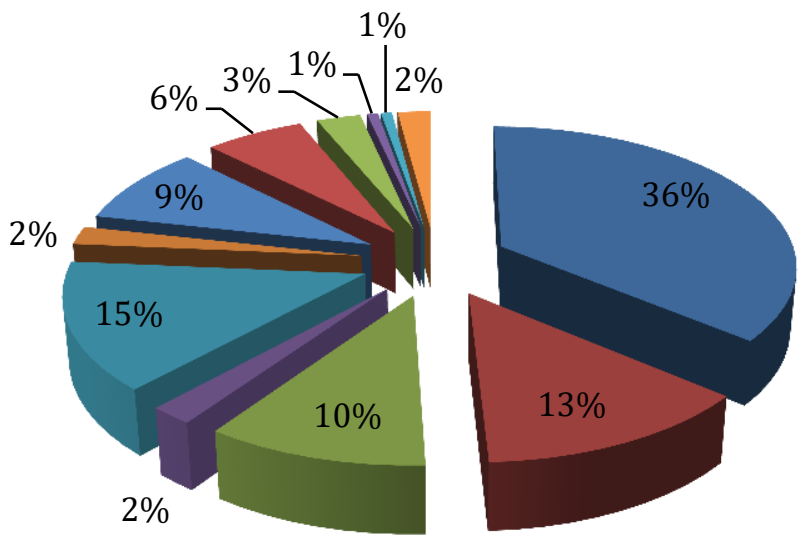

Leaf

Root

Bark

Bulb

Fruit

Whole plant

Seed

Flower

Rhizomes

Water

Partial plant

Gum

Figure 2. Percentages of various plant parts used by local communities

Table 1. Uses of medicinal plants (Trees, shrubs $\&$ herbs) against human diseases

\begin{tabular}{|c|c|c|c|c|c|c|}
\hline $\begin{array}{l}\text { Sl } \\
\text { No }\end{array}$ & Local name & Scientific name & Family & Habit & Part uses & Diseases \\
\hline 1. & Ulot Kambal & Abroma augustum $L$. & Malvaceae & Shrub & $\begin{array}{l}\text { Bark of roots, } \\
\text { Leaf }\end{array}$ & $\begin{array}{l}\text { Ministration disorder, } \\
\text { Gonorrhoea }\end{array}$ \\
\hline 2. & Jhumka & $\begin{array}{l}\text { Abutilon indicum (L.) } \\
\text { Sweet }\end{array}$ & Malvaceae & Shrub & Roots & Early delivery \\
\hline 3. & Apang & Achyranthes aspera L. & Amaranthaceae & Shrub & Roots Plants & Aphordisiac, Arthritis \\
\hline 4. & Basak & Adhatoda vasica Nees & Acanthaceae & Shrub & $\begin{array}{l}\text { Leaves, Bark, } \\
\& \text { roots }\end{array}$ & $\begin{array}{l}\text { Coughing, Reduces high } \\
\text { blood pressure }\end{array}$ \\
\hline 5. & Bel & $\begin{array}{l}\text { Aegle marmelos (L.) } \\
\text { Corrêa }\end{array}$ & Rutaceae & Tree & $\begin{array}{l}\text { Fruits, Leaves, } \\
\text { Roots }\end{array}$ & Constipation \\
\hline 6. & Rashun & Allium sativum $L$ & Amaryllidaceae & Herb & Bulb & Cough, asthma, intestinal \\
\hline 7. & Bishkachu & Alocasia indica Roxb. & Araceae & Herb & Leaves, Roots & $\begin{array}{l}\text { Mouth diseases, Ear } \\
\text { Diseases, }\end{array}$ \\
\hline 8. & $\begin{array}{l}\text { Ghrito } \\
\text { kumari }\end{array}$ & Aloe vera (L.) Burm.f. & Liliaceae & Herb & Leaves extract & $\begin{array}{l}\text { Constipation. In appetite, } \\
\text { Leucorrhoea. }\end{array}$ \\
\hline 9. & Chatim & $\begin{array}{l}\text { Alstonia scholaris (L.) R. } \\
\text { Br. }\end{array}$ & Apocynaceae & Tree & $\begin{array}{l}\text { Bark, Root, } \\
\text { Gum }\end{array}$ & $\begin{array}{l}\text { High blood pressure, } \\
\text { Fever, Nematicide, } \\
\text { Diarrhoea }\end{array}$ \\
\hline 10. & Katanata & Amaranthus spinosus L. & Amaranthaceae & Herb & Roots & Dysentery, Diuretic \\
\hline 11. & Anarash & $\begin{array}{l}\text { Ananas comosus (L.) } \\
\text { Merr. }\end{array}$ & Bromeliaceae & Herb & Leaves, Fruits & $\begin{array}{l}\text { Tape Worm, Urinary } \\
\text { disorder }\end{array}$ \\
\hline 12. & Supari & Areca catechu L. & Arecaceae & Tree & Fruits, leaves & $\begin{array}{l}\text { Stomach disorder, Ulcer, } \\
\text { Diarrhoea }\end{array}$ \\
\hline 13. & Nadana & $\begin{array}{l}\text { Artemisia vulgaris } \\
\text { (Linn.) }\end{array}$ & Asteraceae & Herb & Leaves & ,4clamsia \\
\hline 14. & Shatamuli & $\begin{array}{l}\text { Asparagus racemosus } \\
\text { Willd. }\end{array}$ & Asparagaceae & Herb & Whole plants & $\begin{array}{l}\text { Cancer, Energies, } \\
\text { Stimulator }\end{array}$ \\
\hline 15. & Kamranga & Averrhoa carambola $L$. & Oxalidaceae & Shrub & Fruits, Leave & Coughing, \& Fever \\
\hline 16. & Jatineem & $\begin{array}{l}\text { Azadirachta indica A. } \\
\text { Juss. }\end{array}$ & Meliaceae & Tree & $\begin{array}{l}\text { Bark, Seed, } \\
\text { Leaves, Fruits, } \\
\text { Flowers }\end{array}$ & $\begin{array}{l}\text { Antiseptic, Skin diseases, } \\
\text { Mouth \& Tooth wash }\end{array}$ \\
\hline 17. & Hijal & $\begin{array}{l}\text { Barringtonia acutangula } \\
\text { (L.) Gaertn. }\end{array}$ & Lecythidaceae & Tree & $\begin{array}{l}\text { Leaves, } \\
\text { Branches }\end{array}$ & Cough, Asthma \\
\hline 18. & Shial mutha & $\begin{array}{l}\text { Blumea lacera (Burn.f.) } \\
\text { DC. }\end{array}$ & Asteraceae & Shrub & Root \& Mutha & Dysentery \\
\hline 19. & Punarnava & Boerhavia repens $L$. & Nyctaginaceae & Herb & Leaves & Asthma, Intestinal injury \\
\hline 20. & Shimul & Bombax ceiba $L$. & Malvaceae & Tree & $\begin{array}{l}\text { Bark, Root \& } \\
\text { Seed }\end{array}$ & $\begin{array}{l}\text { Diarrhoea, Dysentery, } \\
\text { Cough removal, Sexual } \\
\text { weakness. }\end{array}$ \\
\hline 21. & Patharkuchi & $\begin{array}{l}\text { Bryophyllum calycinum } \\
\text { Salisb. }\end{array}$ & Crassulaceae & Herb & Leaves & $\begin{array}{l}\text { Kidney stone remover, } \\
\text { Cough \& Fever }\end{array}$ \\
\hline
\end{tabular}




\begin{tabular}{|c|c|c|c|c|c|c|}
\hline $\begin{array}{l}\text { SI } \\
\text { No }\end{array}$ & Local name & Scientific name & Family & Habit & Part uses & Diseases \\
\hline 22. & Palash & $\begin{array}{l}\text { Butea monosperma } \\
\text { (Lam.) Taub }\end{array}$ & Leguminosae & Tree & $\begin{array}{l}\text { Leaves, } \\
\text { Flower, Bark, } \\
\text { Seed }\end{array}$ & $\begin{array}{l}\text { Worm, Bleeding, Cough, } \\
\text { Fever, Menstruation }\end{array}$ \\
\hline 23. & Arohor & Cajanus cajan (L.) Huth & Fabaceae & Shrub & Leaves, Seed & $\begin{array}{l}\text { Hepatitis, Vomiting, } \\
\text { Bronchitis }\end{array}$ \\
\hline 24. & Akanda & $\begin{array}{l}\text { Calotropis gigantea (L.) } \\
\text { W.T. Aiton }\end{array}$ & Apocynaceae & Shrub & $\begin{array}{l}\text { Leaves, Barks } \\
\text { \& Gum }\end{array}$ & $\begin{array}{l}\text { Nauscent, Ruboficient, } \\
\text { Alternative Leprocy, } \\
\text { Asthma }\end{array}$ \\
\hline 25. & Sonalu & Cassia fistula $L$. & Caesalpiniaceae & Tree & Fruits, Leaves & $\begin{array}{l}\text { Constipation, Fungal } \\
\text { infection, Skin diseases }\end{array}$ \\
\hline 26. & Nayantara & $\begin{array}{l}\text { Catharanthus roseus (L.) } \\
\text { G. Don }\end{array}$ & Apocynaceae & Shrub & Root, leaves & $\begin{array}{l}\text { Leukemia, diabetes, } \\
\text { hypertension }\end{array}$ \\
\hline 27. & Telakucha & $\begin{array}{l}\text { Ccecinea cordijolia (L.) } \\
\text { Cogn. }\end{array}$ & Cucurbitaceae & Climber & Leaves & Insanity, Madness \\
\hline 28. & Thankuni & $\begin{array}{l}\text { Centella asisatica (L.) } \\
\text { Urban }\end{array}$ & Apiaceae & Herb & Leaves & $\begin{array}{l}\text { Dysentry, Diarrhoea, } \\
\text { Diseases of urinary tract }\end{array}$ \\
\hline 29. & Tejpata & $\begin{array}{l}\text { Cinnamomum tamala } \\
\text { (Buch.-Ham.) Nees \& } \\
\text { Eberm. }\end{array}$ & Lauraceae & Tree & Leaves \& Bark & $\begin{array}{l}\text { Gonorrhoea, Flavor, } \\
\text { Carmative }\end{array}$ \\
\hline 30. & Lemon & $\begin{array}{l}\text { Citrus aurantifolia } \\
\text { (Cristm.) Swingle }\end{array}$ & Rutaceae & Shrub & $\begin{array}{l}\text { Fruits, \& } \\
\text { Leaves }\end{array}$ & $\begin{array}{l}\text { Vit. C, Digestion, } \\
\text { Appetizer }\end{array}$ \\
\hline 31. & Vat & $\begin{array}{l}\text { Clerodendron } \\
\text { infortunatum Linn. }\end{array}$ & Verbenaceae & Climber & Leaves & $\begin{array}{l}\text { Antianodyne } \\
\text { Anthelmintic }\end{array}$ \\
\hline 32. & Coconut & Cocos nucifera $L$. & Arecaceae & Tree & Water & Diarrhoea, Gastric \\
\hline 33. & Amada & Curcuma amada Roxb. & Zingiberaceae & Herb & Rhizome & $\begin{array}{l}\text { Abdominal \& Gastric } \\
\text { problem, Indigetion }\end{array}$ \\
\hline 34. & Araroot & $\begin{array}{l}\text { Curcuma } \\
\text { angustifolia Roxb. }\end{array}$ & Zingiberaceae & Herb & Tuber & Carminatice \\
\hline 35. & Banhalud & $\begin{array}{l}\text { Curcuma aromatica } \\
\text { Salisb. }\end{array}$ & Zingiberaceae & Herb & Rhizome & $\begin{array}{l}\text { Abdominal, Dycentry \& } \\
\text { Gastric problem }\end{array}$ \\
\hline 36. & Datura & Datura metel L. & Solanaceae & Shrub & Fruits, Leaves & $\begin{array}{l}\text { Pain killer, Asthma. } \\
\text { Mental disorder }\end{array}$ \\
\hline 37. & Krishnachura & $\begin{array}{l}\text { Delonix regia (Hook.) } \\
\text { Raf. }\end{array}$ & Fabaceae & Tree & Flower & Pneumonia, Bleeding \\
\hline 38. & Kendar & $\begin{array}{l}\text { Diospyros tomentosa } \\
\text { Roxb. }\end{array}$ & Ebenaceae & Tree & Fruits & Astringent \\
\hline 39. & Kalasuta & Ehretia ocuminata R.Br. & Boraginaceae & Tree & Plants & Jaundice \\
\hline 40. & Mandar & Erythrina variegata $L$. & Fabaceae & Tree & Leaves, Bark & $\begin{array}{l}\text { Fever, Worm, Meho \& } \\
\text { Gonorrhea }\end{array}$ \\
\hline 41. & Jungle dumur & Ficus racemosa L. & Moraceae & Tree & $\begin{array}{l}\text { Plant juice, } \\
\text { Fruit }\end{array}$ & $\begin{array}{l}\text { Mums, Jaundice, } \\
\text { Dycentery }\end{array}$ \\
\hline 42. & Gandharaj & $\begin{array}{l}\text { Gardenia jasminoides } \\
\text { J.Ellis }\end{array}$ & Rubiaceae & Tree & Flower & Stimulant \\
\hline 43. & Bannalia & $\begin{array}{l}\text { Gelonium multiflorum } \\
\text { A.Juss. }\end{array}$ & Euphorbiaceae & Tree & Leaves & Stimulant \\
\hline 44. & Jaisty madhu & Glycyrrhiza glabra L. & Leguminosae & Climber & $\begin{array}{l}\text { Leaves \& } \\
\text { fruits }\end{array}$ & $\begin{array}{l}\text { Anthelmintic, cold cough } \\
\text { \& asthima }\end{array}$ \\
\hline 45. & Lal Verenda & Jatropha gossypifolia L. & Euphorbiaceae & Shrub & $\begin{array}{l}\text { Seed, Leaf, \& } \\
\text { Roots }\end{array}$ & $\begin{array}{l}\text { Constipation, Rheumatic, } \\
\text { \& Neural weakness }\end{array}$ \\
\hline 46. & Mehedi & Lawsonia inermis $L$. & Lythraceae & Shrub & $\begin{array}{l}\text { Leaves, Barks, } \\
\text { Flowers }\end{array}$ & $\begin{array}{l}\text { Headache, Skin disease, } \\
\text { Antiseptic. Liver } \\
\text { disorder }\end{array}$ \\
\hline 47. & Dandakalash & $\begin{array}{l}\text { Leucas aspera (Roth.) } \\
\text { Spreng }\end{array}$ & Lamiaceae & Herb & Leaves, Seeds & $\begin{array}{l}\text { Labour problem, } \\
\text { Achlamsia }\end{array}$ \\
\hline 48. & Mango & Mangifera indica $L$. & Anacardiaceae & Tree & $\begin{array}{l}\text { Fruits, Leaves, } \\
\text { Seed, Bark, \& } \\
\text { Gum }\end{array}$ & $\begin{array}{l}\text { Diarrhoea, Diabetes. } \\
\text { Asthma, Coughing, Piles. }\end{array}$ \\
\hline 49. & Cassava & $\begin{array}{l}\text { Manihot esculenta } \\
\text { Crantz. }\end{array}$ & Euphorbiaceae & Shrub & Leaves, Bark & Stop bleeding, Pluster \\
\hline 50. & Darharidra & $\begin{array}{l}\text { Morinda angustifolia } \\
\text { Roxb. }\end{array}$ & Rubiaceae & Tree & $\begin{array}{l}\text { Branch, } \\
\text { Leaves }\end{array}$ & Ginolo ical diseases \\
\hline 51. & Shajina & Moringa oleifera Lam. & Moringaceae & Tree & $\begin{array}{l}\text { Berks, Fruits, } \\
\text { Seed }\end{array}$ & $\begin{array}{l}\text { Paralysis, Heart diseases, } \\
\text { Neural weakness, } \\
\text { Historia }\end{array}$ \\
\hline 52. & Tut & Morus indica $L$. & Moraceae & Tree & $\begin{array}{l}\text { Fruits, Bark, } \\
\text { Leaves }\end{array}$ & $\begin{array}{l}\text { Neural disorder, Sex } \\
\text { stimulator, Tape worm. }\end{array}$ \\
\hline
\end{tabular}




\begin{tabular}{|c|c|c|c|c|c|c|}
\hline $\begin{array}{l}\text { Sl } \\
\text { No }\end{array}$ & Local name & Scientific name & Family & Habit & Part uses & Diseases \\
\hline 53. & Atia Kala & Musa sapientum Linn. & Musaceae & Herb & $\begin{array}{l}\text { Roots, Leaves, } \\
\text { \& Fruits }\end{array}$ & $\begin{array}{l}\text { Cholera, Dysentery, } \\
\text { Diarrhoea, Worm, } \\
\text { constipation \& Diabetes }\end{array}$ \\
\hline 54. & Shephali & Nyctanthes arbortristis & Oleaceae & Tree & $\begin{array}{l}\text { Leaves \& } \\
\text { Flower }\end{array}$ & $\begin{array}{l}\text { Malaria, Rheumatic, \& } \\
\text { Worm }\end{array}$ \\
\hline 55. & Lal shapla & $\begin{array}{l}\text { Nymphaea nouchali } \\
\text { Burm.f. }\end{array}$ & Nymphaeaceae & Herb & Flower & Menstrual diseases \\
\hline 56. & Tulsi & Ocimum tenuiflorum L. & Lamiaceae & Shrub & $\begin{array}{l}\text { Leaves, } \\
\text { Flower, seeds, } \\
\text { \& roots }\end{array}$ & $\begin{array}{l}\text { Cough remover, } \\
\text { Bronchitis, Skin diseases, } \\
\& \text { Fever }\end{array}$ \\
\hline 57. & Kanaidinga & $\begin{array}{l}\text { Oroxylum indicum (L.) } \\
\text { Kurz }\end{array}$ & Bignoniaceae & Tree & Leaves & Jaundice \\
\hline 58. & Gandha badali & Paedaria foetida $L$. & Rubiaceae & Climber & Leaves & $\begin{array}{l}\text { Venereal diseases, } \\
\text { Dysentery }\end{array}$ \\
\hline 59. & Khejur & $\begin{array}{l}\text { Phoenix sylvestris (L.) } \\
\text { Roxb. }\end{array}$ & Arecaceae & Tree & Leaves, Roots & Pyorrhea, Stomach \\
\hline 60. & Amloki & Phyllanthus emblica L. & Euphorbiaceae & Tree & Fruits & $\begin{array}{l}\text { Carminative, Vitamin C, } \\
\text { \& Appetizer }\end{array}$ \\
\hline 61. & Chotrapata & Plumbago zeylanica L. & Plumbaginaceae & Herb & Leaves & Antifertility \\
\hline 62. & Raktachandan & $\begin{array}{l}\text { Pterocarpus santalinus } \\
\text { L.f. }\end{array}$ & Fabaceae & Tree & Fruits, Seeds & Skin diseases \\
\hline 63. & Dhalim & Punica granatum $L$. & Punicaceae & Tree & $\begin{array}{l}\text { Stem, Fruits, } \\
\text { \& Roots }\end{array}$ & $\begin{array}{l}\text { Nematicides, Dysentery, } \\
\text { Diarrhoea }\end{array}$ \\
\hline 64. & Sharpagandha & Rauwolfia tetraphylla $L$. & Apocynaceae & Shrub & Leaves & Sedative, Blood pressure \\
\hline 65. & Ashoke & $\begin{array}{l}\text { Saraca asoca (Roxb.) De } \\
\text { Wilde }\end{array}$ & Fabaceae & Tree & $\begin{array}{l}\text { Bark, Leaves } \\
\text { \& Fruits }\end{array}$ & $\begin{array}{l}\text { Ministrations, Piles, } \\
\text { Blood dysentery, Liver } \\
\text { disorder, \& Syphilis. }\end{array}$ \\
\hline 66. & Muchi lat & $\begin{array}{l}\text { Stephania japonica } \\
\text { (Thumb.) Miers }\end{array}$ & Menispermaceae & Climber & Whole plants & $\begin{array}{l}\text { Plaster on injury, } \\
\text { Jaundice }\end{array}$ \\
\hline 67. & Bishkachu & $\begin{array}{l}\text { Steudnera virosa } \\
\text { (Roxb.) Prain }\end{array}$ & Araceae & Herb & Stem & Pain, Gut \\
\hline 68. & Sheora & Streblus asper Lour. & Moraceae & Tree & Leaves & $\begin{array}{l}\text { Diuretic, Dysentery, } \\
\text { Leucrrhoea }\end{array}$ \\
\hline 69. & Chirata & $\begin{array}{l}\text { Swertia chirata Buch.- } \\
\text { Ham. ex Wall }\end{array}$ & Gentianaceae & Herb & Leaves & $\begin{array}{l}\text { Anthelmintic Alergy, } \\
\text { Dycentry, Clean blood }\end{array}$ \\
\hline 70. & Kalojam & $\begin{array}{l}\text { Syzygium cumini (L.) } \\
\text { Skeeels }\end{array}$ & Myrtaceae & Tree & $\begin{array}{l}\text { Roots, Seeds } \\
\text { \& Leaves }\end{array}$ & $\begin{array}{l}\text { Bleeding, Dysentery \& } \\
\text { Diabetes }\end{array}$ \\
\hline 71. & Marigold & Tagetes erecta $L$. & Compositae & Herb & $\begin{array}{l}\text { Leaves, } \\
\text { Flower }\end{array}$ & Anticeptic, Stop bleeding \\
\hline 72. & Arjun & $\begin{array}{l}\text { Terminalia arjuna } \\
\text { (Roxb. ex DC.) Wight \& } \\
\text { Arn }\end{array}$ & Combretaceae & Tree & Bark & $\begin{array}{l}\text { Heart diseases, High } \\
\text { blood pressure, Liver } \\
\text { cirrhosis, Asthma }\end{array}$ \\
\hline 73. & Bohera & $\begin{array}{l}\text { Terminalia belerica } \\
\text { (Gaertn.) Roxb. }\end{array}$ & Combretaceae & Tree & Fruit \& Seed & Hepatitis, Eye Diseases \\
\hline 74. & Katbadam & Terminalia catappa L. & Combretaceae & Tree & Barks \& Fruits & $\begin{array}{l}\text { Constipations, Piles, } \\
\text { Fever \& Menstruation }\end{array}$ \\
\hline 75. & Horitoki & $\begin{array}{l}\text { Terminalia chebula } \\
\text { (Gaertn.) Retz. }\end{array}$ & Combretaceae & Tree & Nut & Stimulant \\
\hline 76. & Amgorancha & $\begin{array}{l}\text { Tinospora cordifolia } \\
\text { (Willd.) Miers }\end{array}$ & Menispermaceae & Climber & $\begin{array}{l}\text { Branch, } \\
\text { Leaves }\end{array}$ & $\begin{array}{l}\text { Antipyretic, Itches, } \\
\text { Maleria }\end{array}$ \\
\hline 77. & Bon piuj & Urginea indica Kunth. & Liliaceae & Herb & Bulb & Dycentry, Antipyretic \\
\hline 78. & Harjora & $\begin{array}{l}\text { Vitis quadrangularis (L.) } \\
\text { Wall. ex Wight \& Arn. }\end{array}$ & Vitaceae & Climber & $\begin{array}{l}\text { Branch, } \\
\text { Leaves }\end{array}$ & $\begin{array}{l}\text { Fracture of bones, } \\
\text { Inappetite }\end{array}$ \\
\hline 79. & Ashwagandha & Withania somnifera (L.) & Solanaceae & Herb & Whole plant & Displacement of Uterus \\
\hline 80. & Banada & $\begin{array}{l}\text { Zingiber purpureum } \\
\text { Roscoe }\end{array}$ & Zingiberaceae & Herb & Rhizome & $\begin{array}{l}\text { Cough, Expectorent, } \\
\text { Stomach ache }\end{array}$ \\
\hline
\end{tabular}

\section{Medicinal plants used against animal diseases}

In the study area 11 medicinal plant species were used against different types of animal diseases by the local people. Highest number of plants were from fabaceae family. Among the total respondents, $12 \%$ were used these species that are given in the following Table 02 . This implied an effective safety net for livestock's with low cost medicines especially for the livestock's of poor people. 
Table 02. Uses of medicinal plants against animal diseases

\begin{tabular}{|c|c|c|c|c|c|c|}
\hline $\begin{array}{l}\text { Sl } \\
\text { No }\end{array}$ & $\begin{array}{l}\text { Local } \\
\text { name }\end{array}$ & Scientific name & Family & Habit & $\begin{array}{l}\text { Part } \\
\text { uses }\end{array}$ & Uses \\
\hline 1. & Bon piaj & Urginea indica Kunth. & Liliaceae & herb & Bulb & Inflamation of throat \\
\hline 2. & Katanata & Amaranthus spinosus $L$. & Amaranthaceae & herb & $\begin{array}{l}\text { Whole } \\
\text { plant }\end{array}$ & $\begin{array}{l}\text { Increase milk content } \\
\text { of cow }\end{array}$ \\
\hline 3. & Anarash & Ananas comosus (L.) Merr. & Bromeliaceae & herb & Leaves & Anthelmintic of cow \\
\hline 4. & Jaina & Fimbristylis littoralis Gaudich. & Cyperaceae & herb & $\begin{array}{l}\text { Whole } \\
\text { plant }\end{array}$ & $\begin{array}{l}\text { Increase digestion } \\
\text { capacity }\end{array}$ \\
\hline 5. & $\begin{array}{l}\text { Jungle } \\
\text { dumur }\end{array}$ & Ficus 1 & Mol & $\mathrm{T}$ & Fruit & $\begin{array}{l}\text { Diarrhoea, cough of } \\
\text { cow }\end{array}$ \\
\hline 6. & Atia Kala & Musa sapientum Linn. & Musaceae & herb & Mutha & $\begin{array}{l}\text { Dysentery, Abdominal } \\
\text { problem of cow }\end{array}$ \\
\hline 7. & Kanta bas & Bambusa bambos (L.) Voss & Poaceae & tree & Leaves & $\begin{array}{l}\text { Solve labour problem } \\
\text { of cow }\end{array}$ \\
\hline 8. & Biskatali & Polygonum lapathifolium $L$. & Polygonaceae & herb & Leaf & Lice \\
\hline 9. & Banhalud & Curcuma aromatica Salisb. & Zingiberaceae & herb & Tuber & Disentry of cow \\
\hline 10. & Banrashun & Cyanotis cristata (L.) D.Don & Zingiberaceae & herb & Bulb & Carminative \\
\hline 11. & Banada & Zingiber purpureum Roscoe & Zingiberaceae & herb & Tuber & Carminative \\
\hline
\end{tabular}

\section{Medicinal plants used for pest control}

The researcher found 10 medicinal plant species which were substantially used against pests of different crops by the $22.67 \%$ of the total respondents. These types of medicinal species with their uses are presented in Table 0 . The findings indicated that there was a possibility of reducing pressure of chemical pesticides by using these types of species. This implied a positive effect on the natural environment if herbal pesticides are promoted.

Table 03. Uses of medicinal plants for pest control

\begin{tabular}{|c|c|c|c|c|c|c|}
\hline $\begin{array}{l}\text { Sl } \\
\text { No. }\end{array}$ & $\begin{array}{l}\text { Local } \\
\text { name }\end{array}$ & Scientific name & Family & Habit & Part uses & Pest control \\
\hline 1. & Jatineem & $\begin{array}{l}\text { Azadirachta indica A. } \\
\text { Juss. }\end{array}$ & Meliaceae & Tree & $\begin{array}{l}\text { Leaves \& } \\
\text { fruits }\end{array}$ & $\begin{array}{l}\text { Stored grain pest, Stem } \\
\text { borer, Aphids, Bugs, Leaf } \\
\text { sucker }\end{array}$ \\
\hline 2. & Datura & Daturc & Solanaceae & Shrub & $\begin{array}{l}\text { Leaves, } \\
\text { Fruits }\end{array}$ & Purple blotch of onion \\
\hline 3. & Cassava & $\begin{array}{l}\text { Manihot esculenta } \\
\text { Crantz. }\end{array}$ & Euphorbiaceae & Shrub & $\begin{array}{l}\text { Young } \\
\text { leaves }\end{array}$ & $\begin{array}{l}\text { Insects were died for } \\
\text { sucking }\end{array}$ \\
\hline 4. & Shajina & Moringa oleifera Lam. & Moringaceae & Tree & Leaves & Stored pests \\
\hline 5. & Tobacco & Nicotiana tabaccum $L$. & Solanaceae & Shrub & Leaves & White fly \\
\hline 6. & Tulsi & Ocimum tenuiflorum $L$. & Lamiaceae & Shrub & Leaves & Aphids \\
\hline 7. & Biskatali & $\begin{array}{l}\text { Polygonum } \\
\text { lapathifolium L. }\end{array}$ & Polygonaceae & Herb & Leaves & $\begin{array}{l}\text { Stored grain pest, Stem } \\
\text { borer }\end{array}$ \\
\hline 8. & Marigold & Tagetes erecta $L$. & Compositae & Herb & $\begin{array}{l}\text { Leaves, } \\
\text { Root }\end{array}$ & Insecticide, Nematocide \\
\hline 9. & Nishinda & Vitex negundo $L$. & Verbenaceae & Herb & Leaves & Majra oka \\
\hline 10. & Banhalud & $\begin{array}{l}\text { Curcuma aromatica } \\
\text { Salisb. }\end{array}$ & Zingiberaceae & Herb & Rhizomes & $\begin{array}{l}\text { Insect pest, Specially for } \\
\text { soil pest }\end{array}$ \\
\hline
\end{tabular}

\section{Conclusion}

The study area was found to be a potential for medicinal plants. Most of the medicinal plant species had diversified uses. Some were used against human diseases, some for livestock diseases and others were used for controlling pests of plants. Although growing medicinal plants and benefits from them have been appeared as a potential enterprise to the concerned households, but several problems such 
as scarcity of land, money inputs etc. were found to be barriers for their commercial cultivation and utilization. Appropriate methods need to be developed to restock natural and domesticated plant supplies of target important species; especially those are rare or endangered. Training and communication for the rural farmers should be arranged for providing necessary information and inputs on identification, usage, cultivation and conservation of medicinal plants.

\section{References}

[1]. Ahmmed M. R., Ahmed, S., Sunny, S. M. S. I., Kar, A., Mahmud, S. M. N., Kabir, M. H., Mahmud, S. and Shaon, S. M. (2017). A study on diversity of medicinal plant usage by folk medicinal practitioners in different villages of Dhunat Upazila, Bogra district, Bangladesh. Journal of Pharmacognosy and Phytochemistry, 6(1), 177-186

[2]. Ahsan, A. T. M. K., Shabuddin, M., Choudhuri, K., Shahriar, M., Huda, A. N. and Choudhury, Z. (1997). Genetic Resources Conservation and Utilization of Medicinal and Aromatic Plants. Plant Genetic Resources Bangladesh prspective. Procedings of a National Work Shop on Plant Genetic Resources. 26-27 August, BARK, Dhaka.

[3]. BARC (Bangladesh Agriculture Research Council). (1972-1992). Flora of Bangladesh. Salar, M. K. (ed.) BARC, Dhaka.

[4]. Chevallier, A. (1996). The Encyclopedia of Medicinal Plants. First edition. DK Publishing Inc., New York.

[5]. Chopra, R. N., Nayar, S. L. and Chopra, I. C. (1992). Glossary of Indian Medicinal Plants. CSIR, New Delhi.

[6]. Chowdhury, J. U., Alam, M. K. and Hasan, M. A. (1996). Some Traditional Folk Formularies Against Dysentery and Diarrhoea in Bangladesh. Journal of economic and taxonomic botany, 12: 20-23.

[7]. Das, K. D. and Alam, M. K. (2001). Trees of Bangladesh. Bangladesh Forest Research Institute, Chittagong.

[8]. Ghani, A. (2003). Medicinal Plants of Bangladesh with Chemical Constituents and Uses. Second edition. Asiatic Society of Bangladesh, Ramna, Bangladesh

[9]. Hannah, L. and Bowles, I. (1995). Letters, global properties. Bioscience. pp. 45-122. https://doi.org/10.2307/1312547

[10]. Miah, M. D. and Chowdhury, M. S. H. (2003). Indigenous healthcare practice through medicinal plants from forests by the Mro tribe in Bandarban region, Bangladesh, INDILINGA- African Journal of Indigenous Knowledge Systems, 2(2), 61-73.

[11]. Rahman, M. (2003). Science World: Veshog Udvider Rokomfer (in Bangla). Proffessor's Prokashon, Dhaka. June 2003, p. 46.

[12]. Rashid, M. H., Alam, M. J., Ara, R. and Merry, S. R. (1987). Crude Drug Market Survey, Bangladesh. Bangladesh Forest Research Institute, Chittagong (Unpublished).

[13]. Roy, P. (2001). The Daily Bhorer Kagaz: Bangladesher Biponno Jeeb Boichitro 6 (in Bangla). December, pp. 1-2.

[14]. Silva, T. D. (1997). Industrial Utilization of Medicinal Plants in Developing Countries. Nonwood forest products-11, Medicinal plants for forest conservation and health care, FAO, Rome, Italy, p.34.

[15]. Srivastava, J., Lambert, J. and Vietmeyer, N. (1995). Medicinal plants: An expanding Role in Development. World Bank. Agriculture and Forestry Systems, Washington, D.C.

[16]. Walden, I. (1995). Preserving Biodiversity: The role of property rights. In: Swanson, T. Ed. Intellectual Property Rights and Biodiversity Conservation: An Interdisciplinary Analysis of the Values of Medicinal Plants. Cambridge University Press, UK. 176-198. https://doi.org/10.1017/CB09780511623417.009

[17]. Zohora Z. T., Mahmud, S. M. N., Kar, A., Mahmud, S and Shibly, A. Z. (2016) A Survey on medicinal plant usage by folk medicinal practitioners in different villages of Haluaghat upazila, Mymensingh District, Bangladesh, Indian Journal of Research in Pharmacy and Biotechnology, 4(5), 200-208. 